\title{
4. ÉPHÉMÉRIDES (EPHEMERIDES)
}

PRÉSIDENT: J. Kovalevsky.

Vice-PrÉSIDENT: R. L. Duncombe.

Comité d'Organisation: V. K. Abalakin, W. Fricke, A. M. Sinzi, G. A. Wilkins.

\section{INTRODUCTION}

Le présent rapport diffèrera sensiblement des précédents rapports de la Commission 4 publiés à l'occasion des précédentes assemblées générales de l'UAI. Pendant longtemps, en effet, les activités correspondant au domaine couvert par la Commission étaient concentrées dans les services nationaux et internationaux des éphémérides. Mais désormais, de nouveaux groupes, souvent en liaison avec les organismes engagés dans la Recherche Spatiale, contribuent de façon essentielle à l'amélioration du système de constantes fondamentales et construisent des éphémérides de grande précision pour les corps du système solaire. C'est pourquoi, $j$ 'ai préféré examiner les activités de recherche dans ce domaine de façon globale, indépendamment des organismes ayant subventionné ces recherches.

Ayant ainsi regroupé ailleurs des renseignements donnés dans les rapports des Directeurs des services des Éphémérides, il n'était plus possible (faute de place et afin d'éviter toute duplication) de publier in-extenso les rapports. Dans ces conditions, il a paru plus logique d'analyser aussi de façon globale l'activité de ces services et de supprimer totalement la traditionnelle annexe réservée à leurs rapports.

\section{BUREAU INTERNATIONAL D'INFORMATION SUR LES ÉPHÉMÉRIDES ASTRONOMIQUES}

Le Bureau International d'Information sur les Éphémérides Astronomiques (BIIEA) a commencé à travailler sous la direction de B. Morando. Nous donnons ci-après son compte-rendu d'activité.

"The International Information Bureau on Astronomical Ephemerides, established by the XIVth General Assembly of the IAU, commenced its operation in 1971 and is located at the Bureau des Longitudes in Paris. The scientific advisory committee, including representatives of IAU and COSPAR met twice and established the procedures to follow.

Fifty information cards have been issued in 1972 and sent to 135 organizations. It is expected that about 80 new cards will be issued before the next General Assembly and that about 40 new organizations will be added to the distribution list. Financial support from the International Astronomical Union and Bureau des Longitudes have made this operation possible and is gratefully acknowledged".

Signed by: R. L. DUNCOMBE

Chairman of the Scientific Advisory Committee

B. MORANDO

Director of the BIIEA.

\section{GROUPES DE TRAVAIL DE LA COMMISSION}

Trois groupes de travail avaient été créés par la Commission 4 pour étudier de nouvelles réformes à apporter au système de constantes fondamentales (précession, nutation, masses des planètes), les problèmes posés par ces réformes et l'éventualité d'une redéfinition de l'échelle de temps servant de base aux éphémérides. Deux d'entre eux n'ont commencé leur activité que relativement tard, 
mais présenteront néanmoins leurs conclusions pour la 15ème Assemblée Générale de l'UAI à Sidney. En revanche, le groupe de travail sur les unités et les échelles de temps a procédé, par correspondance, à de nombreux échanges de vues et, bien qu'aucun accord général n'ait été atteint, une synthèse du travail a pû être établie par son président, G. A. Wilkins.

(a) Groupe de travail sur les constantes de la précession

Membres: Fricke (Président), Lederle (secrétaire), Aoki, Clube, Kovalevsky, Lieske, Lundquist, Nemiro, Seidelmann, Vasilevskis et Vicente.

Un compte-rendu est donné en annexe 1.

(b) Groupe de travail sur les éphémérides des planètes

Membres: Duncombe (Président), Janiczek (secrétaire), Abalakin, Herrick, Klepczynski, Kovalevsky, Morando, Oesterwinter, O'Handley, Schubart et Sinclair.

Un rapport sera préparé pour Mars 1973.

(c) Groupe de travail sur les unités et les échelles de temps

Membres: Wilkins (Président), Morrison (secrétaire), Chebotarev, Clemence, Cook, Guinot, Kovalevsky, Shapiro et Van Flandern.

On trouvera le rapport de ce groupe en annexe 2.

\section{LE SYSTÈME UAI DE CONSTANTES FONDAMENTALES}

C'est en 1964 que l'Union Astronomique Internationale a adopté ce système et recommandait qu'il soit progressivement introduit, pour autant que cela sera possible à partir de 1968, dans les Éphémérides Nationales et Internationales (UAI Transactions, Vol. XIIB, p. 105). En 1972, il est possible de dresser le bilan de cette introduction: on peut considérer, en effet, que le processus a été achevé et que le contenu de ces ouvrages est stabilisé pour plusieurs années. Dans toute la mesure où cela était possible sans avoir à refaire complètement la théorie du mouvement de certains corps, toutes les Éphémérides Nationales et Internationales sont établies dans le système UAI de constantes fondamentales, du moins dans les limites de la précision avec laquelle les tableaux sont publiés.

Plusieurs cas se présentent:

(1) Les positions apparentes des étoiles et les constantes pour la réduction au jour sont entièrement calculées dans le nouveau système.

(2) Les éphémérides des satellites des planètes et les éphémérides pour les observations physiques des planètes, publiées avec une faible précision, sont pratiquement inchangées, sinon pour la modification de la valeur de la constante d'aberration.

(3) Les éphémérides de la Lune publiées sont désormais directement calculées dans le système UAI à partir de la théorie de Brown améliorée (Éphéméride $j=2$ d'après la définition de 1967 de l'UAI). Il en est de même des prévisions d'éclipses et d'occultations.

(4) Les éphémérides des planètes sont calculées avec toutes les constantes UAI à l'exception de la masse du système Terre-Lune. En effet, ces théories n'ont pas été refaites suivant une recommandation de l'UAI (Trans. IAU, Vol. XIIIB, p. 4-7). Les erreurs ainsi commises pour les grosses planètes sont négligeables par rapport à la précision des tabulations. Pour le Soleil, Mercure, Venus, Mars et les petites planètes, ces erreurs peuvent atteindre quelques centièmes de seconde de degré et sont sensibles pour celles des éphémérides qui donnent 0"01 (1e Soleil dans la plupart des Éphémérides).

\section{SYSTÈME DE MASSES PLANÉTAIRES}

Le problème de la construction d'un nouveau système de masses planétaires, qui avait été laissé inchangé en 1964 lorsque l'on a défini le système UAI de constantes astronomiques, est toujours à l'ordre du jour et est étudié par le Groupe de travail, dirigé par R. L. Duncombe, établi à cet 
effet en 1970. Cette perspective a encouragé les recherches pour l'amélioration des masses des planètes extérieures, qui sont encore inaccessibles aux méthodes spatiales et qui, dans le système présent, sont les plus incertaines.

A l'U.S. Naval Observatory, ces recherches ont particulièrement porté sur la détermination de la masse de Jupiter par l'étude du mouvement des petites planètes, notamment Freia (1), Cybèle (2), Pales (3), Mnemosyne (4) et Polyhymnia (5). Au Bureau des Longitudes, on obtenait un résultat comparable avec le IXème satellite de Jupiter (6), tandis qu'à l'Astronomisches Rechen-Institut, des déterminations analogues étaient obtenues à l'aide de Hilda, Thulé et Chicago (7).

De nouvelles déterminations de la masse de Saturne et Uranus ont été faites à l'U.S. Naval Observatory par l'étude des perturbations planétaires (8) et les résultats ont été bien confirmés par des solutions générales du mouvement des planètes obtenues par le Lincoln Laboratory (9). Une nouvelle amélioration des masses de Jupiter et Mercure est à prévoir à la suite des missions de Pioneer 10 vers Jupiter et de la sonde Mercury vers Venus et Mercure en 1973, alors qu'il semble qu'il y ait une limitation assez sevère quant à la précision que l'on peut atteindre par la méthode des perturbations planétaires (10).

Plusieurs tentatives ont été faites pour proposer un système cohérent de masses planétaires d'apres les récentes déterminations, aussi bien à l'U.S. Naval Observatory (11) qu'au Bureau des Longitudes (12), travaux qui font suite à ceux qui ont été présentés par des équipes de ces Établissements et du JPL au Colloque UAI No. 9 (voir Celestial Mechanics, Vol. 4, No. 2).

\section{BIBLIOG R A PHIE}

1. Klepczynski, W. J., Seidelmann, P. K. and Duncombe, R. L. 1970 Astron. J., 75, 739.

2. O'Handley, D. A. $\quad 1970$, Astron. Papers Am. Ephem., 20, pt. III.

3. Doggett, L. E. 1971, Astron. J., 76, 486.

4. Fiala, A. 1972, Astron. Papers Am. Ephem., 21, pt. II.

5. Janiczek, P. M. 1972, Astron. Papers Am. Ephem., 21, pt. I.

6. Polavieja, M. G. de, et Edelman, C. 1972, Astr. and Astrophys., 16, 66.

7. Scholl, H. 1971, Celestial Mechanics, 4, 250.

8. Klepczynski, W. J., Seidelmann, P. K. and Duncombe, R. L. $\quad 1970$, Astron. J., 75, 739.

9. Ash, M. E., Shapiro, I. I. and Smith, W. B. 1971, Science, 174, 551.

10. Seidelmann, P. K. 1972, Celestial Mechanics, $5,3$.

11. Duncombe, R. L., Klepczynski, W. J. and Seidelmann, P. K. 1973, 'The Masses of the Planets, Satellites and Asteroids', Fundamentals of Cosmic Physics (in press).

12. Kovalevsky, J. 1973, 'A System of Planetary Masses and Related Quantities', Phys. Earth Planet. Interiors, Vol. 6 (in press).

\section{RECHERCHES SUR LA CONSTANTE DE LA PRÉCESSION \\ ET LE CATALOGUE FONDAMENTAL}

Ces recherches ont été menées de façon active à l'Astronomisches Rechen-Institut, comme le montre la communication suivante de W. Fricke.

"Newcomb's determination of precession has been rediscussed by Fricke (1) in an effort to ascertain the reason for the deficiencies in Newcomb's results. Newcomb's basic material consists of the Bradley Stars included in Auwers' catalogue. Fricke has analysed the proper motions of a sample of 265 Bradley stars, which are at distances greater than about $100 \mathrm{pc}$ and are included in the proper motion systems of Auwers, Newcomb, and FK4. A solution based on Newcomb's system and neglecting galactic rotation has confirmed Newcomb's result within the prevailing accuracy. Other solutions for the same stars based on the system of FK4 have shown that Newcomb's neglect of galactic rotation explains part of the deviation from recent determination. The main part is explained by deficiencies in Newcomb's and Auwers' proper motion systems.

On the basis of improved values for the planetary masses Laubscher (2) has derived the corrections $+00^{\prime \prime} 036 \pm 0 \prime 006$ and $-0{ }^{\prime} 029 \pm 0 \prime 010$ per century at 1850.0 to Newcomb's values of the secular 